\title{
Plasma shape stabilisation of current rise MHD instabilities in TCV
}

\author{
A. Scarabosio $\S$, A. Pochelon and Y. Martin \\ Ecole Polytechnique Fédérale de Lausanne (EPFL) \\ Centre de Recherches en Physique des Plasmas \\ Association Euratom-Confédération Suisse \\ CH-1015 Lausanne, Switzerland \\ E-mail: andrea.scarabosio@ipp.mpg.de
}

\begin{abstract}
The well-known and potentially disruptive plasma "current rise" instabilities are studied as a function of the plasma shape in the tokamak $\grave{a}$ Configuration Variable (TCV). Disruptions typically occur in quasi-circular plasmas at $q_{a} \sim 3$ in both non-sawtoothing and sawtoothing discharges with peaked current profiles. The perturbations in the plasma parameters before disruption are characterised, and the main unstable modes identified as coupled $m / n=2 / 1$ and $3 / 2$ rotating tearing modes. In the early phase, coupling between $3 / 1$ and $2 / 1$ modes is found to play a major role in determining whether or not the disruption will occur. Plasma cross section shaping is observed to reduce or to completely stabilise the disruptive mode and is regularly used in TCV operation as a tool for safe initial current ramp-up. Plasma elongation, positive and negative triangularity prevent the growth of a large $2 / 1$ mode at $q_{a} \sim 3$, thus reducing or even suppressing the disruptions. We also attempt an interpretation of the experimental results. Calculations of the tearing mode stability parameter $\Delta^{\prime}$ using the experimental plasma equilibria suggest the dominant role of toroidal mode coupling in the destabilisation of the $m / n=2 / 1$ mode in quasi-circular TCV plasmas. The effect of shaping on the reconstructed current profile and tearing stability is then considered. The analysis shows a destabilising trend with elongation and triangularity in contrast with the experiment. Other stabilising mechanisms are discussed and shown to potentially contribute to the safe crossing of $q_{a}=3$ in shaped plasmas.
\end{abstract}

\section{Introduction to plasma instabilities during current rise}

The abrupt termination of a plasma discharge, often called plasma disruption, is of major concern in present day and future tokamak fusion experiments. A disruption causes, in fact, high heat and mechanical stresses to the structures surrounding the plasma column (the vessel wall, the divertor, the magnetic coils, the diagnostics, etc.). In large devices, such as fusion reactors, these stresses can provoke serious damages. Disruptions need $\S$ present address: Max-Planck Institut für Plasmaphysik, IPP-EURATOM Association, Garching, Germany. 
to be avoided or mitigated. Understanding the dynamics leading to plasma disruption, and developing techniques for avoiding it, are therefore crucial to a tokamak reactor and important parts of today's research in fusion science.

MHD instabilities and disruptions are commonly observed during the plasma current rise phase of tokamaks [1, 2, 3, 4]. The MHD mode activity is sharply correlated with the edge safety factor $q_{a}$. These modes appear as bursts of magnetic activity localised at the plasma edge, where surface kink modes are expected, and when at or just above a rational $q_{a}$ value. The magnetic perturbation shows $n=1$ and $m=q_{a}$ mode numbers, typically associated with low $q(q=5,4,3,5 / 2)$ rational surfaces. Although these surface modes are too localised to induce a disruption, under certain conditions they are accompanied by other instabilities, as the $m=2$ modes, which can grow to large amplitude, lock to the external conducting wall, and finally lead to plasma disruption.

It is generally agreed that large, growing magnetic islands can cause plasma disruption by inducing ergodization of the field lines, followed by a rapid loss of confinement (thermal quench), and eventually by plasma current decay [1]. The Tearing Mode (TM) instability is known to be responsible for the formation of the magnetic islands observed in tokamaks.

In circular plasma cross-section machines, such as TCA, LT-4, FT and Tore-Supra tokamak [2, 3, 4, 5], large $m / n=2 / 1$ tearing modes are reported to reduce the accessibility to low- $q_{a}$ plasmas. Narrow windows for stable trajectories in the $\left(q_{a}, n_{e}\right)$ plane are found around $q_{a} \sim 3$ during the initial current and density rise. These instabilities are generally attributed to TM-unstable current profile partly induced by the rapid current rise rate. In the low- $\beta$ regime (classical TM), in fact, the TM stability mainly depends on the current density profile [6]. Current profiles in stationary ohmic plasmas are typically peaked owing to the strong coupling with the electron temperature through plasma resistivity. During the current rise phase, the current profile can be different from its fully relaxed (stationary) shape due to the skin effect. When the current rise time is shorter than the current redistribution time, the current profile can be flat or hollow in the centre with possibly large gradients toward the edge [3]. In general, large gradients in the current profile may destabilise MHD modes. Current profiles with large edge gradients may be unstable with respect to the external-kink modes and/or to tearing modes. Therefore, the formation of peaked current profile seems to be crucial for the creation of stable plasmas at low- $q$. The idea of an improved stability of peaked current profile with $q_{0}<1$ is corroborated by the easier crossing of $q_{a}=3$ in sawtoothing discharges as observed in some experiments. Low density is also favourable, since it favours centrally peaked current profile.

In large shaped tokamaks, such as JET, for instance, it is usually sufficient to decrease the current ramp rate in order to peak up the current profile, keeping the MHD activity at a sufficiently low level, and thus avoiding disruptions [1]. In the circular tokamak Tore Supra, obtaining stable discharges with $q_{a}<3$ is made difficult by the presence of large MHD modes appearing during the current rise phase [5] even at small current rise rate. The generation of an "ergodic divertor" (layer of ergodic magnetic 


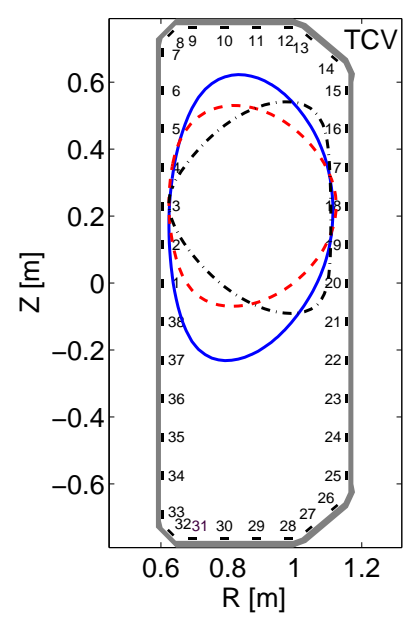

Figure 1. Poloidal magnetic probes location and different TCV plasma shapes used in this study: quasi-circular (\#21400 $\kappa=1.3 \delta=0.2$, dashed line), moderately shaped with negative triangularity (\#21402 $\kappa=1.3 \delta=-0.4$, dashed-dotted line), and mediumhigh elongated plasma (\#21399 $\kappa=1.7 \delta=0.2$, solid line). The position of the poloidal Mirnov coils are indicated (38 probes). The toroidal arrays of 16 low field side (LFS) and 8 high field side (HFS) probes are located at the height of the probes 3 and 18.

field at the plasma edge) was reported to improve the plasma stability allowing stable discharges with $q_{a}=2.5$. In this article we show that the plasma current of quasicircular plasmas in the TCV tokamak is often limited by disruptions at $q_{a} \sim 3$ also in discharges with centrally peaked current profile. To find a stable path toward a plasma with safety factor values below 3 , a suitable control of the plasma shape is required during the current rise.

In section 2 of this paper we describe the main experimental findings. These include the characterisation of the MHD modes responsible for the disruptions during the current rise at $q_{a} \sim 3$ in Ohmic TCV plasmas and show the beneficial effect of plasma shaping leading to a dramatic reduction of the disruptivity. We also show experimental evidence of the destabilising coupling between a tearing mode and an external-kink mode, which is thought to trigger for the growth of large magnetic islands. In section 3, we attempt an interpretation of the observations. We first analyse the stability of the experimental current profiles in quasi-circular plasmas against the $2 / 1$ mode with and without taking into account the toroidal coupling. We then consider the effects of plasma shaping on the equilibrium current profile and tearing stability suggesting mechanisms for the observed stabilisation. Conclusions are finally drawn in section 4.

\section{Experimental observations}

The Tokamak à Configuration Variable (TCV) [7] has a standard aspect ratio $1 / \epsilon=$ $R_{0} / a \sim 3.5$ with $R_{0}=0.88 \mathrm{~m}, a \leqslant 0.25 \mathrm{~m}, I_{p} \leq 1 \mathrm{MA}, B_{\phi, 0}<1.54 \mathrm{~T}$. The TCV design allows maximal flexibility in producing different plasma shapes and magnetic configurations, its aim being the study of the effects of plasma shaping and configuration 
on plasma stability and transport. The flexibility in plasma shaping is achieved by the combination of 16 independently controlled poloidal shaping coils and a strongly elongated vacuum vessel $\kappa_{T C V}=2.9$ (figure 1), which is almost entirely covered by graphite titles.

The current rise instability is studied in pure ohmically heated L-mode discharges limited by the central column with different values of the elongation $\kappa$ and triangularity $\delta$. In a typical experiment, the plasma shape is kept constant while the current is raised by properly adjusting the loop voltage $\left(V_{\text {loop }}<3 \mathrm{~V}\right)$. As a consequence, the edge safety factor decreases in time at a controlled rate. To explore the effect of plasma shaping, the elongation is varied on a shot-to-shot basis from 1.1 to 1.8 , and the triangularity from -0.5 to +0.4 . In TCV, increasing the plasma density is found to reduce $l_{i}$, to enlarge the current density profile, and thus to influence the stability of current driven modes. For this reason, the gas valve is pre-programmed to maintain a constant central line integrated electron density at a value of typically $\bar{n}_{e}=3 \cdot 10^{19} \mathrm{~m}^{-2}$ throughout these experiments. However, the strong dependence between the plasma current and the ionisation rate, leads to a density increase during the current rise (figure 2). The density variation is kept within $10 \%$ during the relevant part of the current ramp, i.e. between $q_{a}=4$ and $q_{a}=3$. This small variation is believed to only slightly affect the current profile and the plasma stability properties.

In a few discharges the electron density was varied in the range $\bar{n}_{e}=2-5 \cdot 10^{19} \mathrm{~m}^{-2}$, always on a shot-to-shot basis. Within this range of variation, the effect of plasma density on this type of disruptions has only a second order effect with respect to the plasma shaping and will be here neglected.

In order to simplify the discussion, the plasmas in these experiments can be divided in three categories according to their level of shaping: strongly shaped $(\kappa>1.7$, or $\delta>0.3$ or $\delta<-0.4)$, moderately shaped $(1.7>\kappa>1.4,-0.4<\delta<-0.3$ or $0.2<\delta<0.3$, quasi-circular plasmas $(\kappa<1.4,-0.3<\delta<0.2)$. Some examples of TCV plasma shapes used in this study are shown in figure 1 .

The standard initial current rise rate in TCV is rather high $\left(d I_{p} / d t=2 \mathrm{MA} / \mathrm{s}\right)$, and may lead to transient flat or hollow current profile. A set of experiments with slower current rise has therefore been performed to study specifically the precursors disruption activity (figure 2).

Note that a sufficiently slow current ramp up is an essential condition for these experiments, since it allows for:

- a complete current penetration with the production of quasi-stationary centrally peaked current profiles typical of ohmic discharges where the skin effect can thus be neglected.

- the extension of the disruption precursors duration, allowing for a better analysis of the perturbations.

To achieve these conditions the time scale of the current rise, $\tau_{I_{p}}=I_{p} /\left(d I_{p} / d t\right) \sim$ $500 \mathrm{~ms}$, is made longer than the current diffusion time, $\tau_{\eta} \sim 100 \mathrm{~ms}$, as calculated 


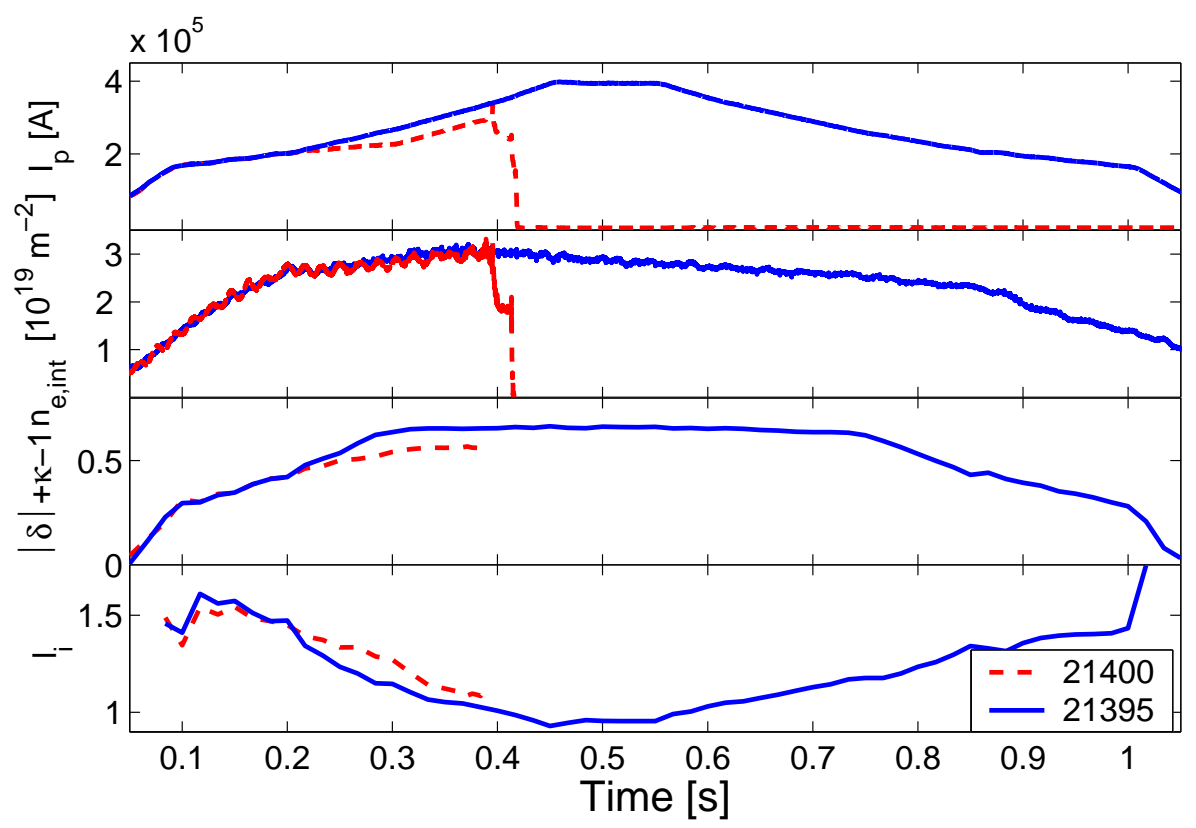

Figure 2. Evolution of the plasma current, electron density, a shape factor defined (arbitrarily) as $|\delta|+\kappa-1$, internal inductance $l_{i}$ and edge safety factor during typical current ramp up experiment in a disruptive (dashed line) and non-disruptive (full line) discharge.

from typical experimental profiles. The equilibrium reconstruction, provided by the code LIUQE [8], is based on external magnetic measurements (with no internal current profile measurements). The presence of sawtooth oscillations visible in the soft Xray emission indicates a central safety factor smaller than 1 , in agreement with the results of the LIUQE code, confirming the presence of a peaked current profile. The
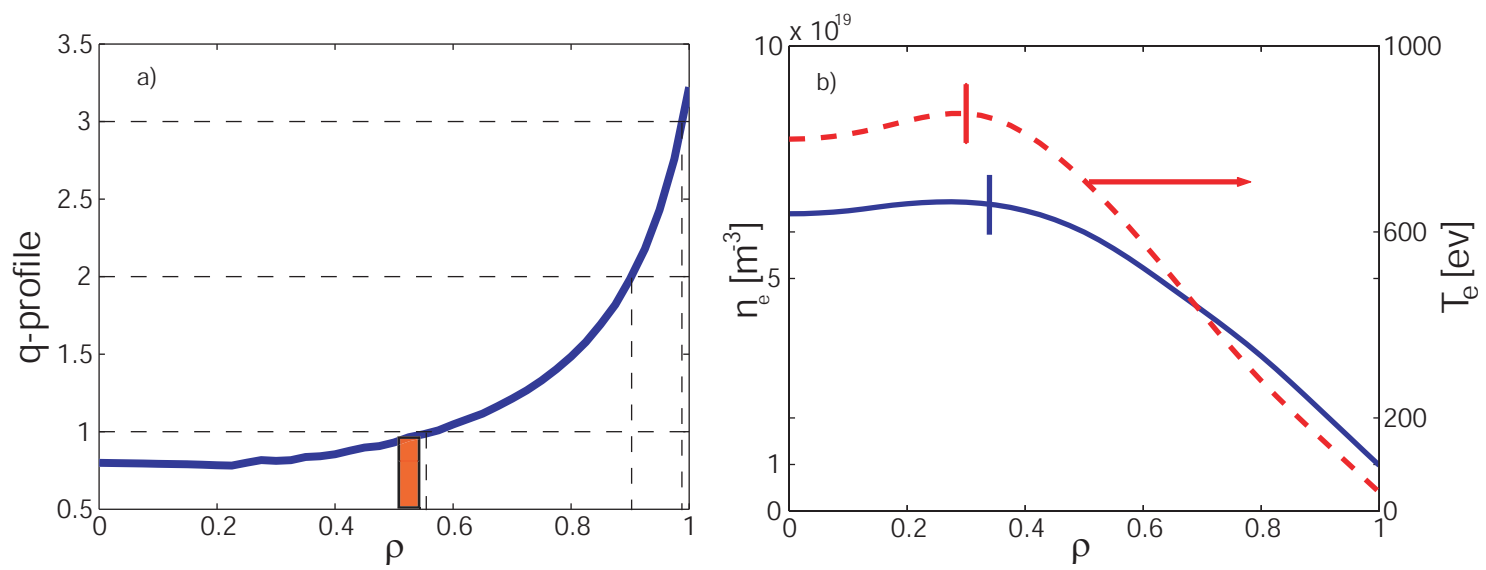

Figure 3. a) LIUQE reconstruction of the $q$ profile at $\mathrm{t}=0.367 \mathrm{~s}$, approximately at the time of modes appearance (discharge $\# 21400, \kappa=1.32, \delta=0.2$, in Fig. 2). The estimation of the sawtooth inversion radius from soft X-ray tomography is shown in red. b) electron density and temperature fitted profiles from Thomson scattering.

LIUQE experimental quasi-stationary current profiles are also found to be consistent 


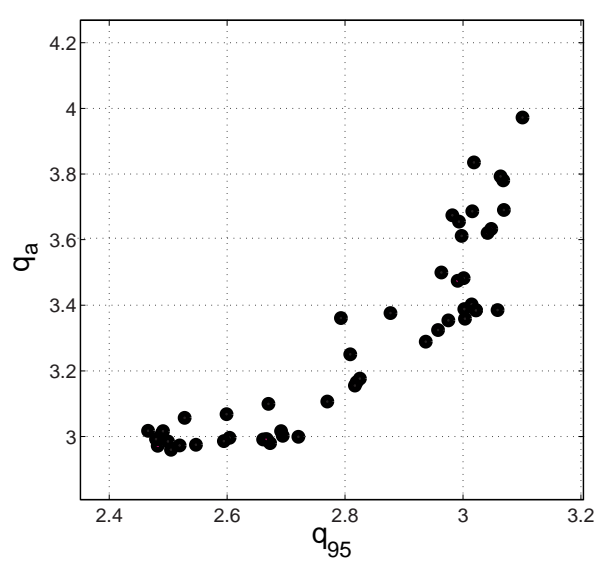

Figure 4. Values of $q_{a}$ and $q_{95}$ at the first appearance of MHD activity during the current ramp-up for discharges of all types of shape. At the mode appearance, the $q=3$ surface is located inside the plasma but close the edge, generally in the last $5 \%$ of the poloidal magnetic flux.

with numerical simulations of the experimental discharges using the fixed boundary transport code PRETOR [9]. The PRETOR code can simulate the discharge evolution with and without the inclusion of the current diffusion equation and with the neoclassical resistivity calculated from experimental profiles. These simulations show that significant transient modifications of the current profile are induced for current rise rates larger than $1 \mathrm{MA} / \mathrm{s}$, whereas the experimental values do not exceed $0.7 \mathrm{MA} / \mathrm{s}$. Here the safety factor is defined as,

$$
q(\rho)=\frac{d \psi_{\text {tor }}}{d \psi_{\text {pol }}}
$$

where $\psi_{\text {tor }}$ and $\psi_{\text {pol }}$ are respectively the toroidal and poloidal magnetic flux and $\rho$ is the squareroot of the normalised poloidal flux. An example of the reconstructed $q$-profile is shown in figure 3 a . The electron density and temperature profile from the Thomson scattering diagnostic prior to the disruption are typically peaked off axis and flat inside $q=1$, as shown in figure $3 \mathrm{~b}$.

\subsection{MHD activity in quasi-circular plasmas with $q_{a} \sim 3$ during current rise}

To reconstruct the structure of the precursors, the magnetic fluctuation, $\delta B$, are measured with poloidal field probes installed inside the vacuum vessel. The probes are arranged in poloidal and toroidal arrays and can resolve low to medium poloidal and toroidal mode numbers $m$ and $n$. The toroidal array on the outboard mid-plane consists of 16 equidistantly spaced probes located, for this study, at a height of $Z=23 \mathrm{~cm}$, close to the equator of the discharge, and resolves the toroidal numbers $n=0, \ldots 8$. The poloidal mode number can be obtained from magnetic measurements with 38 poloidally distributed pick-up coils, which surround the whole poloidal circumference (figure 1). The interpretation of the poloidal mode structure, in terms of components with index $m$ of a Fourier decomposition in the poloidal angle, is complicated by the high elongation 


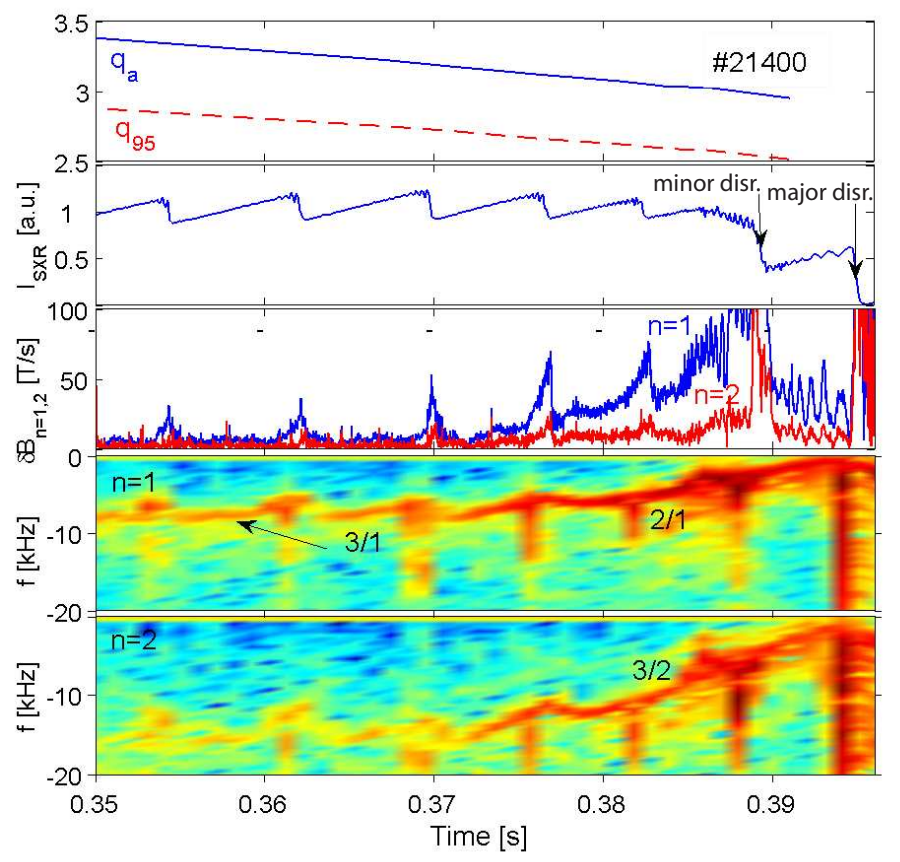

Figure 5. Disruptive instability during the current rise at $q_{a} \sim 3$ in a weakly shaped plasma $(\kappa=1.3, \delta=0.2)$. From top to bottom, $q_{a}$ and $q_{95}$, the central soft X-ray emissivity showing sawtooth activity, the amplitude of the $n=1$ and $n=2$ toroidal components of the magnetic perturbation and the spectrogram of the $n=1$ and $n=2$ magnetic signal (with indication of the mode numbers $m / n$ ).

of the vacuum vessel, the toroidal geometry and the great flexibility in plasma shape that couple and distort the Fourier components. The experimental mode structure is retrieved using a singular value decomposition (SVD) of the full set of toroidal and poloidal magnetic measurements and compared with a model of the magnetic perturbations. The model is based on force- and divergence-free current perturbations flowing along the equilibrium magnetic field lines on a resonant surface $q=m / n$ [10, 11] (helical perturbations leading to the formation of magnetic islands), which allow for the calculation of the magnetic field at the location of the probes taking into account eddy currents on the vacuum vessel. A comparison of phase and amplitude of the modelled and measured mode structure then allows the identification of the unstable mode.

After the crossing of the $q_{a}=4$, the first detectable MHD instability normally appears when the $q=3$ magnetic surface enters the last $5 \%$ of the poloidal magnetic flux, equivalent to $q_{95}<3$ (see figure 4) while the disruption typically occurs at $q_{a}<3$. It is worth stressing this result because it links the precursors activity and the disruptions with the presence of the $q=3$ flux surface close to the plasma boundary. Clearly, this situation has a negative influence on the plasma stability.

An example of the evolution of the MHD activity in a disruptive discharge is shown in figure 5. Owing to the mutual interaction of modes resonant on several rational surfaces, and to the influence of the external conductive wall, the evolution of the 
disruptive instability is rather complex.

We focus here on the pre-disruption MHD activity of a weakly shaped discharge $(\kappa=1.3, \delta=0.2)$, which can be divided in four stages:

(i) An MHD mode of dominant toroidal number $n=1$ typically starts when the $q=3$ rational surface moves toward the edge region $(\mathrm{t}=0.355 \mathrm{~s}$ in fig. 5$)$. The mode rotates in the electron diamagnetic drift direction with a frequency in the range $5-15 \mathrm{kHz}(\sim 7 \mathrm{kHz}$ in the example of figure 5) and initially has an $m=3$ dominant poloidal number. The mode amplitude and frequency are modulated by the sawtooth crashes.

(ii) As the current ramp-up continues, the mode amplitude increases, changing the dominant poloidal number from $m=3$ to $m=2$. The mode frequency decreases.

(iii) When the mode amplitude is in the range $\delta B_{n=1}=0.5-2 \mathrm{mT}$, an $m / n=3 / 2$ mode is triggered. The mode frequency keeps decreasing. Typically we have $\omega_{n=2}=2 \omega_{n=1}$, corresponding to perturbations located on the $q=2$ and $q=1.5$ rational flux surfaces, locked in phase and rotating at the same speed. Note that, near the disruption, the $3 / 2$ mode is considered as a secondary mode, generally too localised in the plasma core to induce alone the disruption [3].

(iv) Depending on the particular discharge, one or more rapid collapses (minor disruptions), associated with a fast growth of the $n=1$ and $n=2$ mode components can precede the major disruption. A quasi-circular discharge often disrupts at $q_{a} \sim 3$, although in some cases it may survive despite the presence of the large MHD mode and reach $q_{a} \sim 2.5$ before finally disrupting.

The measured poloidal mode structure is found in good agreement with a modeled coupled 2/1 and 3/2 magnetic island (figure 6). The two islands are locked with their two X-points at the low field side (LFS) resulting in a constructive interaction and larger total amplitude at the LFS edge. The tearing mode is assumed to be responsible for the formation of the magnetic islands. A cylindrical estimate [12] of the island widths at the time of the disruption indicates comparably large $2 / 1$ and $3 / 2$ island sizes of 5 and $7 \mathrm{~cm}$ respectively and radially overlapping, thus suggesting that magnetic field stochastisation may be an essential mechanism responsible for the rapid loss of confinement. Moreover the presence of the $1 / 1$ (sawtooth) and 3/1 components possibly extends the stochastic region up to the plasma limiter. Interestingly, the modelling of the edge magnetic structure indicates that the 3/1 component does not grow to a large amplitude but remains at least three or four times smaller than the other components of the perturbation, thus suggesting a secondary role in the late disruption phase.

The "current rise disruption" in TCV shows several of the most common disruption features observed in tokamak devices. For instance, mode locking to the vacuum vessel [13] or the simultaneous presence of coupled resistive modes are common in the predisruption MHD activity of "density limit disruption" and "low- $q$ disruption" [1, 2].

The destabilisation of the secondary $3 / 2$ mode in presence of a large $2 / 1$ TM was also observed in other tokamaks [3], and has been the subject of theoretical investigation 

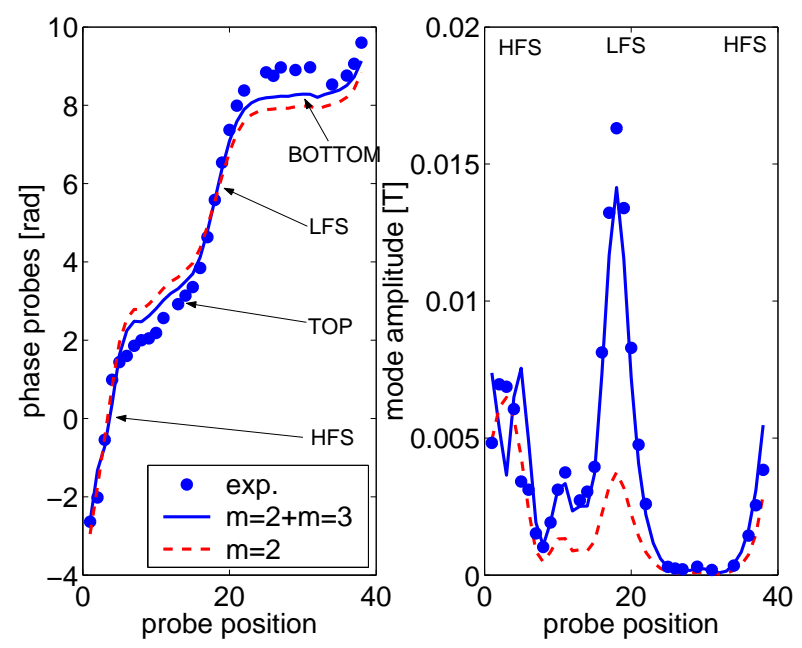

Figure 6. Comparison of the poloidal magnetic structure from SVD analysis of the pre-disruption mode and magnetic island model in discharge \#21400 at $\mathrm{t}=0.378 \mathrm{~s}$. The magnetic probes are numbered clockwise from the HFS to the LFS (see fig. 1). The phase of the mode is dominated by the $m=2$ component (dashed line) but good agreement on both phase and amplitude is only obtained using the two poloidal harmonics $m=2$ and $m=3$, resonant on $q=2$ and $q=1.5$ respectively (full lines).

involving nonlinear effects. Reference [14, for example, invokes the modification of the current profile due to the simultaneous presence of the $2 / 1$ and $1 / 1$ islands. By enhancing the radial thermal transport, the modes flatten the electron temperature and current profiles within the $1 / 1$ and $2 / 1$ magnetic islands. Since the total current is kept constant (unlike the total plasma energy content) by a loop voltage feed-back, the local current gradients in the region between the $q=1$ and $q=2$ surfaces increase and destabilise the tearing mode on the $q=3 / 2$ surface. Another well-known non-linear mechanism thought to be responsible for mode destabilisation is the so-called 'three-wave coupling', described for example in [15], which may also contribute to the destabilisation of this mode. In this article, however, we are mainly interested in the role of the $2 / 1$ mode, in its coupling to the $3 / 1$ mode during the pre-disruption phase and in the change of its stability properties with the plasma shape. In TCV, we find that this type of disruptions can generally be avoided by shaping the plasma cross-section before reaching $q_{a} \sim 3$, as described in the next section.

\subsection{Avoidance of the $q=3$ current rise disruption by means of plasma shaping}

The beneficial effect of plasma shaping in suppressing MHD activity and disruption at the $q=3$ crossing was noticed early in TCV operation. During the initial current rise phase, a sufficiently large plasma elongation or triangularity allows for a safe crossing of the $q=3$ rational surface through the plasma boundary.

In the $\kappa$-scan in figure 7 the maximum amplitude of the $n=1$ component decreases abruptly at an elongation of $\kappa \sim 1.35$, passing from a dominant $m=2$ to $m=3$ poloidal harmonic. Without the formation of a large $2 / 1$ mode, the disruption is avoided. 
In the same way, plasma triangularity is also able to reduce the MHD activity and suppress the growth of the 2/1 mode. Notably, both positive and negative values of triangularity improve the MHD stability and help avoiding plasma disruptions. A similar beneficial effect of the triangularity on the stability of the internal kink mode, responsible for sawtooth oscillations, has already been noticed in TCV plasmas [16, 17].
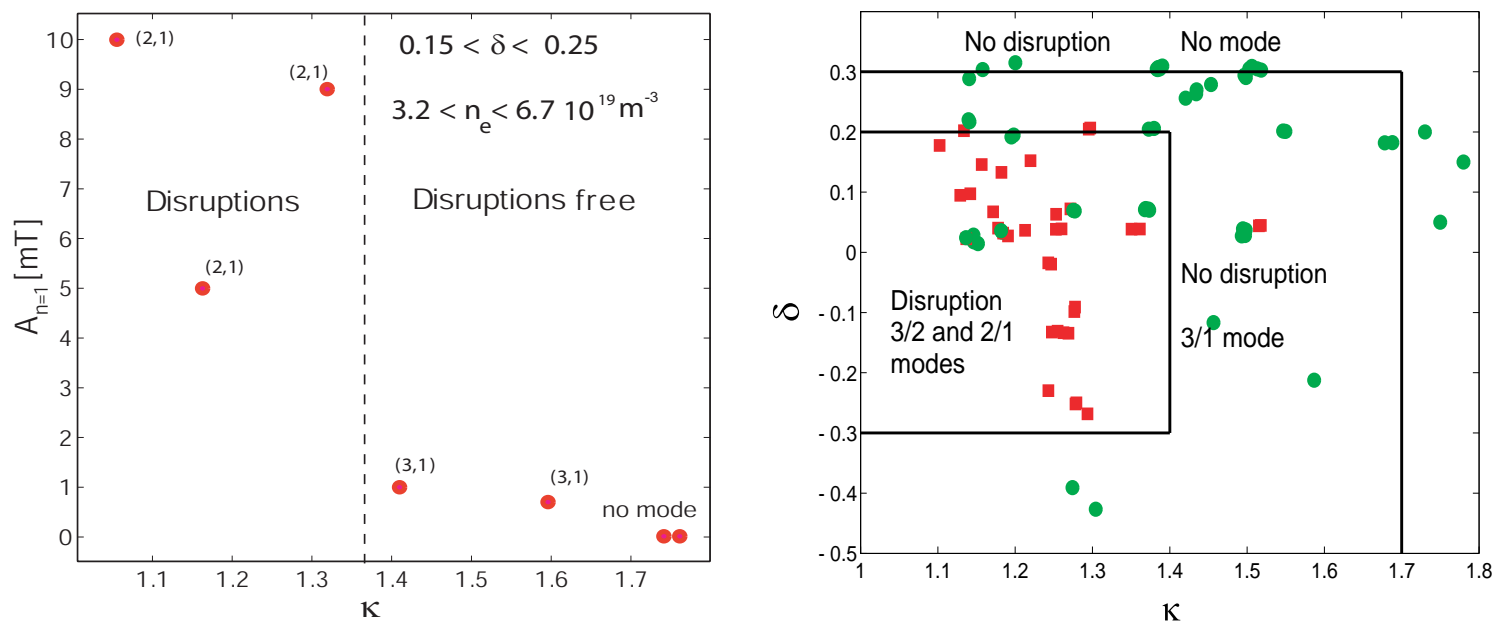

Figure 7. Left: Amplitude of the $n=1$ MHD activity as a function of the plasma elongation $\kappa$. The dominant mode numbers are also indicated. Large elongation values strongly reduce the risk of disruptions during current rise by stabilising the $m / n=2 / 1$ tearing mode. Right: disruption events in the $\kappa-\delta$ plane. Dots represent non disruptive discharges whereas red dots represent disruptive discharges.

The effect of plasma shaping is schematised in figure 7, where the $\kappa-\delta$ space has been divided in disruptive and non-disruptive regions. This graph is useful to show the effect of plasma shaping on $q=3$ disruptions. It should be kept in mind, however, that the boundaries between stable and unstable regions are only indicative due to the limited number of discharges considered. Also, the possible effect of other variables such as the plasma density, current ramp-up rate and the effect of the profiles have not been taken into account.

In quasi-circular plasmas, disruptions occur when the edge safety factor decreases below 3 , due to the presence of a large $2 / 1$ magnetic island as described in section 2.1. In moderately shaped plasmas, the magnetic signal shows transient bursts of MHD activity not leading to plasma disruption. The $3 / 2$ mode remains stable and the $2 / 1$ mode is either stable or saturates at small amplitude, without any coupling observed. This shown in in figure 8 where the MHD activity of a moderately shaped discharge with $\kappa=1.25$ and $\delta=-0.4$ reveals a $3 / 1$ mode growing in amplitude with the frequency abruptly decreasing, while the $2 / 1$ mode remains stable, allowing the current ramp-up to be continued without disruption. We also observe that instabilities with dominant mode numbers $m / n=4 / 1,3 / 1$ and $5 / 2$ are detected when respectively the $q_{a}=4,3$ and 2.5 surfaces are crossed, indicating the destabilising influence of the external kink 
mode when a low order rational surface approaches the plasma boundary, but are not accompanied, in this mildly shaped discharge, by mode locking. In strongly shaped plasmas the MHD activity remains below the detectable level and, remarkably, even the 3/1 mode remains below detectable level.

To summarise, moderate plasma shaping acts primarily on the stability of internal modes (mainly $m / n=2 / 1$ ) and, at large values of elongation and triangularity, leads also to the stabilisation of the external kink mode.

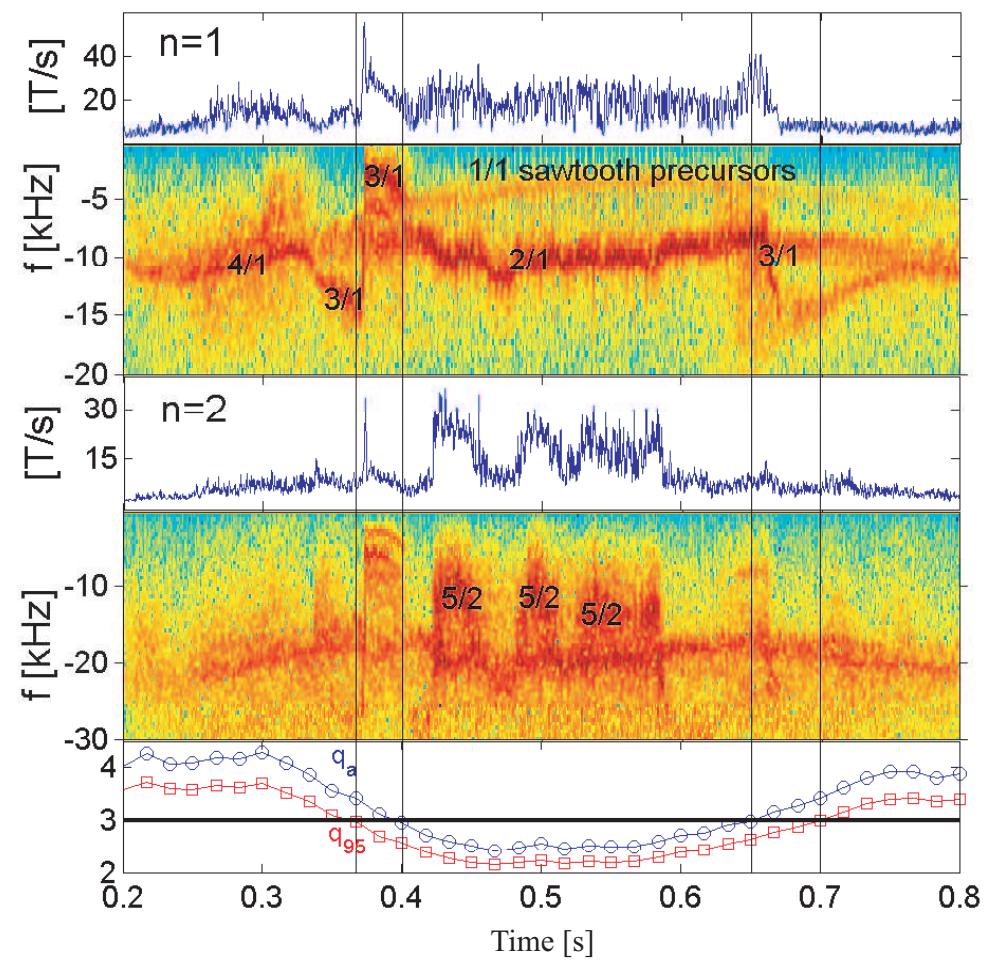

Figure 8. Current ramp-up experiment (\#21402) at negative triangularity $(\delta=-0.4)$. Amplitude and spectrogram of the $n=1$ and $n=2$ toroidal components of the magnetic perturbation are shown. Negative frequencies correspond to mode rotation in the electron diamagnetic drift direction. The temporal evolution of the safety factors at the edge and at $95 \%$ of the poloidal flux shows the crossing of the $q=3$ surface through the plasma boundary.

\subsection{Evidence of the destabilising coupling between the $m / n=3 / 1$ and $m / n=2 / 1$} modes

The peculiarity of the current rise disruption lays, perhaps, in the early stage of the disruption precursors, where the interaction of the $3 / 1$ and $2 / 1$ modes plays an important role in the global stability and evolution toward the disruption. In TCV, it is clearly observed that a $3 / 1$ mode precedes the fast growth of the $2 / 1$ tearing mode $(t=0.370$ $\mathrm{s}$ in figure 5 ). This $3 / 1$ surface mode is believed to be closely related to the ideal MHD external kink mode, which tends to be unstable when a low order rational surface approaches the plasma edge [1, 18]. In these experimental conditions, the external kink 


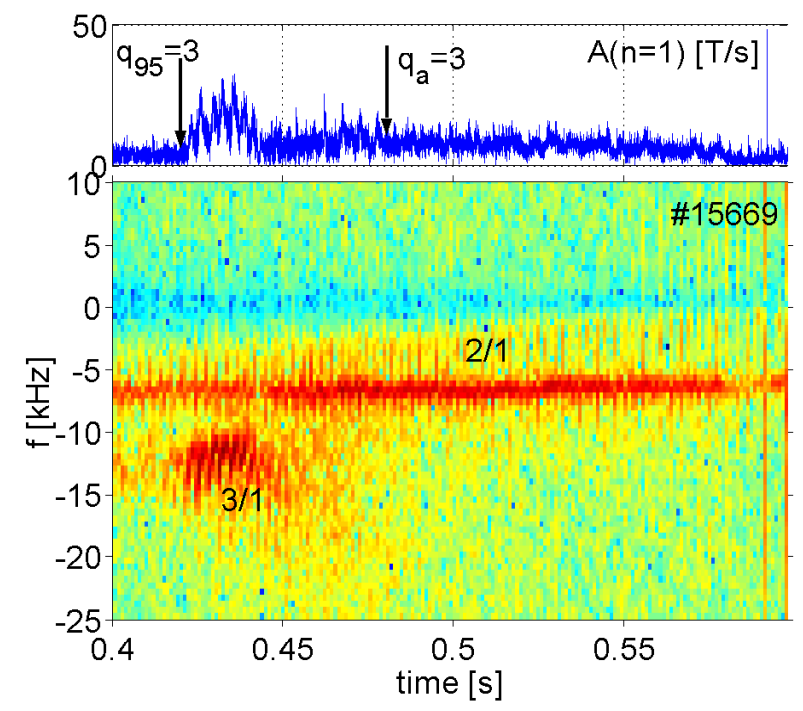

Figure 9. Toroidal $n=1$ component of the magnetic signal (top) and relative spectrogram (bottom) in a quasi-circular ( $\kappa=1.2, \delta=0.2)$ non disruptive current ramp-up discharge. The $3 / 1$ mode is destabilised at $q_{95}=3$, saturates while rotating in the electron diamagnetic drift direction (negative frequency) at 12-13 kHz, and finally disappears after about $30 \mathrm{~ms}$. The 2/1 mode rotates at $7 \mathrm{kHz}$, saturates at small amplitudes and disappears at $t=0.58 \mathrm{~s}$.

is localised at the plasma surface and, according to the magnetic data, does not grow to a large amplitude. It is thus rather harmless in itself (see also section 2.2), but may nevertheless couple to the $q=2$ flux surface and lead to the destabilisation of the $m=2$ magnetic island [19, 20]. Owing to the electromagnetic force, the perturbations of two rotating coupled modes tend to lock in phase, independently of their initial natural frequency. The "natural" frequency of an MHD mode depends, in general, on the equilibrium $\vec{E} \times \vec{B}$ and diamagnetic flows. When current perturbations resonant on different rational surfaces are locked in phase they can interact resonantly. If resonant interaction occurs, this leads to a further destabilisation of the mode, then increasing the linear growth rate [21] and saturation level [20]. We present here experimental evidence of the important role of mode coupling in driving large islands.

The FFT analysis of the $n=1$ component of the disruption precursors clearly shows a single frequency $\omega_{n=1}(t)$ with no discontinuities (see spectrogram in figure 5), while at the same time the dominant poloidal mode number continuously changes from $m=3$ to $m=2$. This indicates that the modes, located on the $q=2$ and $q=3$ surfaces, are locked since the early stage of the precursors activity. The locked perturbations, acting resonantly, grow rapidly, leading to the disruption. This is to be compared with the evolution of discharges where mode coupling does not occur. In that case the $3 / 1$ and $2 / 1$ modes coexist, for instance, but uncoupled, rotating at different frequencies. Such an example is shown in figure 9, where the $3 / 1$ mode rotates at about $12 \mathrm{kHz}$, and the $2 / 1$ mode at $7 \mathrm{kHz}$, thus showing from the different rotation frequencies. The $2 / 1$ mode saturates at small amplitude and eventually disappears without causing any disruption. 
It can be noted that the $3 / 1$ mode is temporarily destabilised when $q_{95}$ goes below 3 (top plot in figure 9), as often observed in similar experiments [1]. Before the $q=3$ surface leaves the plasma (at $t=0.48 \mathrm{~s}$ ), the $3 / 1$ mode vanishes while the $2 / 1$ mode persists at small amplitude. The saturation at small amplitude of the $2 / 1$ mode may be interpreted as the result of the absence of mode coupling with the external surface mode, which appears to be a key element of the dynamic in this type of disruptions.

It should be noted that the conditions for mode locking depends strongly on the natural mode frequencies [21, 22] that are linked to the details of the plasma equilibrium such as pressure and toroidal rotation profile. A slightly larger differential rotation may, at least temporarily, prevent locking and thus allow crossing $q_{a}=3$. This may explain the absence of locking between modes in the discharge in figure 9, which is otherwise similar to other disruptive quasi-circular discharges (i.e. the discharge in figure 5).

\section{Tearing mode stability in TCV shaped plasmas at $q_{a} \sim 3$}

In the following, we attempt to explain why quasi-circular TCV plasmas during the current ramp are subject to large instabilities and disruptions, and why these instabilities are suppressed by plasma shaping. At this purpose, we adopt the linear MHD theory of resistive modes. Consequently, we limit our analysis to the mode onset, leaving the non-linear growth that leads to disruption to future studies.

In purely ohmic discharges in TCV, the plasma pressure is sufficiently low $\left(\beta_{p}=\right.$ $0.1-0.4)$ to allow neglecting the bootstrap current. Thus, the tearing mode is considered to be in the so-called "classical" regime. According to the linear resistive MHD theory with negligible mode coupling, the stability criterion of a classical tearing mode is given by [6],

$$
\Delta^{\prime} \equiv \lim _{\delta \rightarrow 0} \frac{1}{\Psi\left(r_{s}\right)}\left[\frac{d \Psi}{d r}\left(r_{s}+\delta\right)-\frac{d \Psi}{d r}\left(r_{s}-\delta\right)\right]<0
$$

with $\Psi=r B_{r}$ and $r_{s}$ is the radius of the resonant surface. To study the tearing stability, the free energy parameter $\Delta^{\prime}$ has in general to be evaluated numerically including all toroidal, shape and pressure effects. In section 2.1 we showed that the $2 / 1$ tearing mode is the dominant instability leading to disruptions. We will therefore focus our analysis on this mode.

A qualitative understanding of the $2 / 1$ stability can be obtained with the widely used large-aspect-ratio, low- $\beta$ MHD model in reference [18]. This model uses a parabolic current profile of the form $j(r)=j_{0}\left(1-(r / a)^{2}\right)^{\nu}$ with $\nu=q_{a} / q_{0}-1$ and $a$ the minor radius. In cylindrical geometry this current profile corresponds to the $q$-profile $q(r)=q_{0} r^{2} /\left(1-\left(1-r^{2}\right)^{\nu+1}\right)$ with $q_{0}=2 B_{0} / \mu_{0} R_{0} j_{0}$. The plasma stability is usually described in terms of the edge safety factor $q_{a}$ and the current profile peaking $q_{a} / q_{0}$, which are free parameters. The $\left(q_{a} / q_{0}, q_{a}\right)$ plane can be divided in stable and unstable regions as in figure 10 . The region of the $\left(q_{a} / q_{0}, q_{a}\right)$ plane where $2 / 1$ tearing modes may be expected is delimited by the $q_{0}=2$ and $q_{0} \sim 0.5$ black full lines. The trajectories in the $\left(q_{a} / q_{0}, q_{a}\right)$ plane of four discharges with different plasma shaping are also drawn (see 


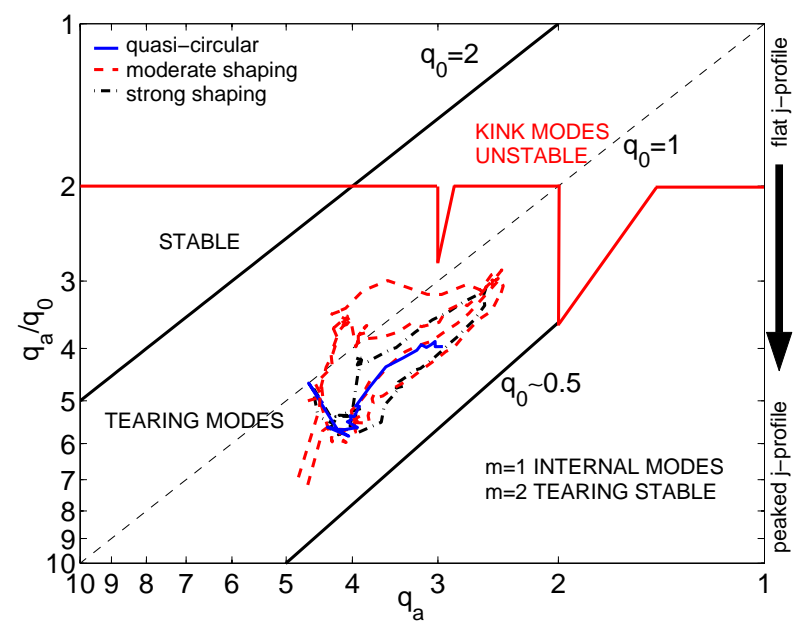

Figure 10. Large aspect ratio and low-beta MHD stability diagram for current profiles $j(r)=j_{0}\left(1-(r / a)^{2}\right)^{\nu}$ (redrawn from fig. 35 of reference [18]). The trajectory in the $\left(q_{a} / q_{0}, q_{a}\right)$ plane of four discharges with different plasma shape are also drawn (see also table (1).

table 1 for the other parameters of the discharges in figure 10). The four trajectories lay well inside the region unstable to tearing modes despite the fact that only the quasi-circular discharge disrupt (full line). It should be noted, however, that the actual TM stability depends on the details of the current (and pressure) profile, and being in the unstable region of this diagram is not sufficient for the instability to occur. More precise indications on plasma stability can be obtained by a detailed analysis making use of experimentally reconstructed equilibria, toroidal geometry and plasma cross section shape.

The resistive stability code PEST-3 [23] calculates the $\Delta^{\prime}$ from the ratio of the Frobenius expansion coefficients of the small and large solutions of the marginal MHD stability equation in full toroidal geometry, which are then matched from each side of the rational surface $s$. Here the Mercier stability index $\mu \equiv \sqrt{-D_{I}}$ is required to be real (locally, on the resonant surfaces under consideration) to satisfy the requirements for ideal stability and the calculation of the tearing stability index. The numerical method implemented in the code is well suited for shaped plasmas with finite pressure and low $m$ and $n$ mode numbers. Note that PEST-3 has already been used to compare the predicted tearing stability with the experimental MHD activity for a variety of plasmas with low and high $\beta$ and $q_{\min }$ [24, 25]. From these studies it was concluded that the stability analysis with PEST-3, in combination with an accurate equilibrium reconstruction, was sufficient to explain the destabilisation of the classical 2/1 tearing mode observed in DIII-D current ramp experiments.

Here, we compare the predictive capability of $\Delta^{\prime}$ calculations with PEST-3 and the onset of tearing modes, typically observed in the TCV current ramp experiments with quasi-circular plasmas. For a first comparison, we neglect the effect of mode coupling and we use equation 2 as the criterion for the linear stability of the mode. 


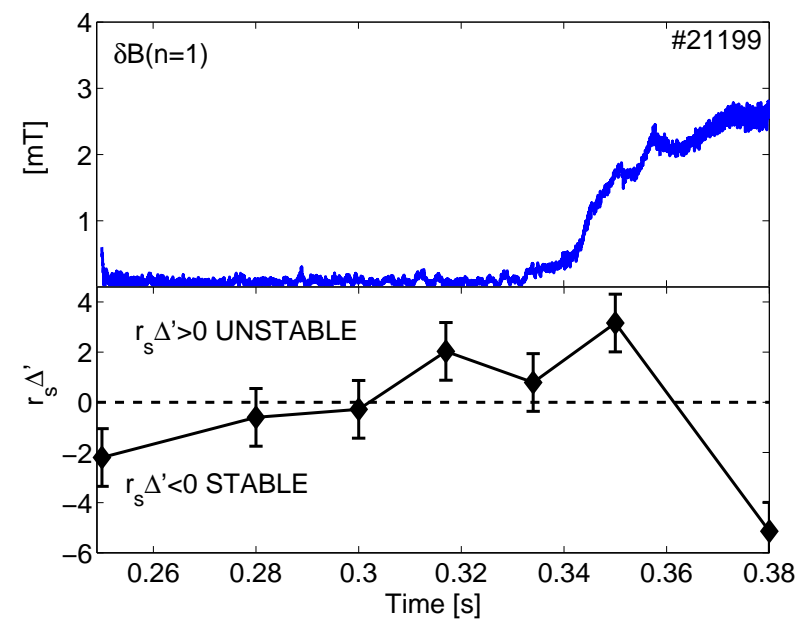

Figure 11. Time trace of the MHD $n=1$ magnetic perturbation (top) and $\Delta^{\prime}$ stability results from PEST-3 (bottom) during a current rise, disruptive, discharge with "low shaping" $(\kappa \simeq 1.25$ and $\delta \simeq 0.05)$ and flat profile $\left(q_{a} / q_{0}=3.5\right)$ close to the onset of a $2 / 1$ mode. The positive value of the $\Delta^{\prime}$ indicates an unstable classical tearing mode. The error bars are based on the sensitivity of $\Delta^{\prime}$ on small changes in the equilibrium, obtained by varying free parameters in the LIUQE code.

We find that, except in few cases apparently linked with the presence of a very flat current profile, the $2 / 1$ mode is stable during the current ramp up even though a large mode was detected. Since the plasma shape is kept constant, the edge safety factor decreases monotonically during the current rise (see figure 2) and the current profile tends to flatten. In cases where the current profile flattens sufficiently (ex. faster ramps), the theoretical predictions and the experiment are in reasonable agreement given the uncertainty (figure 11). In most discharges with large MHD modes and disruptions, however, $\Delta^{\prime}$ is negative (stable tearing) during the ramp. A typical example is shown in figure 12 (see also figures 3 and 5 ).

For these plasmas, "standard" tearing-mode theory with no mode coupling (equation 2) fails to explain the experiments and other mechanisms have to be found to account for the presence of modes.

It should be mentioned that the current density is not directly measured in TCV. We argue, however, that the current density profile is sufficiently well reconstructed for these ohmic discharges with slow ramps. The equilibria used here are not only consistent with magnetic flux measurements, but the reconstructed $q$-profiles are also found, a posteriori, to be consistent with the time of sawteeth appearance/disappearance and with the value of the sawtooth inversion radius, which constrains the position the $q=1$ surface. Other local features of the current profile may not be resolved, but they are unlikely able to explain the inconsistency between the $\Delta^{\prime}$ calculations and the experimental behaviour of tearing modes in TCV.

Mode coupling, which is so far not included in the analysis, has been often invoked to explain the destabilisation of modes [1]. Under certain conditions the theory of 
coupled tearing modes predicts a strong destabilisation of the $2 / 1$ tearing mode due to toroidal coupling [21]. We consider the effect of toroidal coupling for the TCV case in the next section.

\subsection{Toroidal destabilisation of the tearing mode}

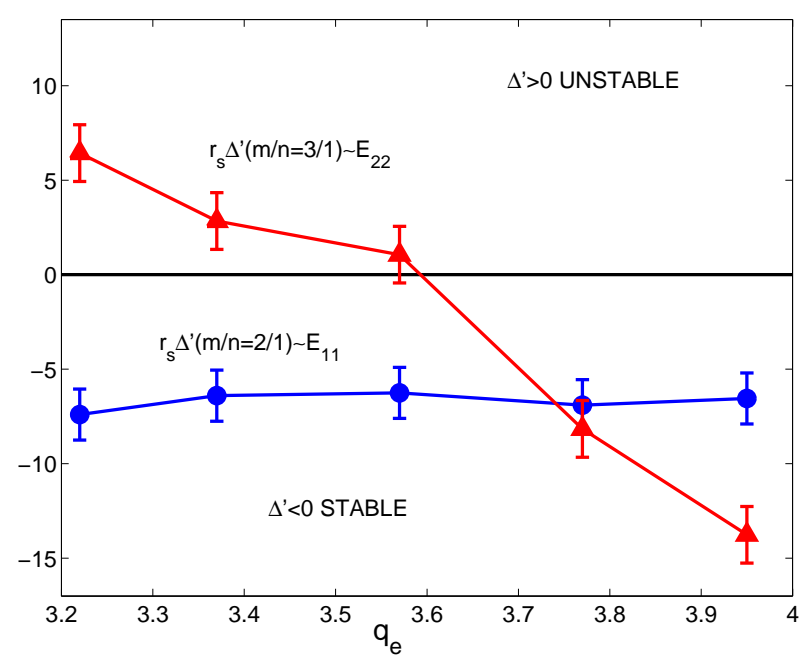

Figure 12. Tearing stability diagram for $m=2$ mode (circles) and $m=3$ mode (upward triangles) in a current rise, disruptive, discharge with "low shaping" $(\kappa \simeq 1.3$ and $\delta \simeq 0.2)$ and peaked current profile $\left(q_{a} / q_{0}=4.0\right)$. Negative (positive) $\Delta^{\prime}$ values correspond to stable (unstable) modes. The error bars are based on the sensitivity of $\Delta^{\prime}$ on small changes in the equilibrium, obtained by varying free parameters in the LIUQE code. Discharge \#21400.

In a toroidal plasma the different poloidal harmonics are coupled by the geometry. To the lowest order, the main harmonic $m$ drives, through toroidicity, the poloidal sidebands $m \pm 1$ resonant on other flux surfaces.

Following the linear theory of coupled modes in references [22, 21], let us consider two resonant flux surfaces $q=m / n$ and $q=m+1 / n$, namely the $q=2$ and $q=3$ surfaces. Let also $\omega_{1}$ and $\omega_{2}$ be the "natural" rotation frequencies of the tearing modes at the $q=2$ and $q=3$ surfaces respectively. The dispersion relation of two coupled rotating tearing modes has, in general, two complex roots for the eigenvalue $\omega$. A particular solution of the dispersion relation can be expressed as:

$$
\begin{aligned}
& \omega=\omega_{1}+i \frac{E_{22}}{\tau_{2}} \\
& \omega=\omega_{1}+i \frac{1}{\tau_{1}}\left[E_{11}-\frac{E_{12}^{2}}{E_{22}}\right] .
\end{aligned}
$$

The diagonal elements $E_{i i}$ obtained by solving the ideal MHD equations correspond, in the cylindrical limit, to the tearing mode stability index $E_{i i}=r_{s_{i}} \Delta_{i}^{\prime}$. The offdiagonal coupling coefficient $E_{12}$ typically increases with the inverse aspect ratio $a / R$ and with decreasing distance between the coupled surfaces (note that in the high magnetic 
shear edge region the rational surfaces are close to each other and thus coupling is strong). A positive imaginary part of $\omega$ corresponds to an unstable mode. The solutions of the tearing dispersion relation in equations 3 and 4 assume the following conditions: (i) the linear response of the resistive layer at each resonant surface $j$ given by $r_{s} \Delta^{\prime}=-i\left(\omega-\omega_{j}\right) \tau_{j}$ where $\tau_{j}$ is the local reconnection time scale; this expression is thought to be appropriate for ohmically heated tokamak plasmas and is often referred as to the visco-resistive regime; (ii) the mode rotation frequency are the same $\left(\omega_{1} \sim \omega_{2}\right)$; in this case, the interaction between modes is maximised since the associated current perturbations are resonant; note that this condition is well satisfied in the described experiments (see section 2.1); (iii) the reconnection times are very different from each other $\tau_{1} \gg \tau_{2}$ (i.e. when one surface is close the plasma edge, as the $q=3$ surface in the present case).

It has been shown in reference [22] that, under these conditions, the inner mode may become more unstable than in the absence of resonant coupling. Equation 4, in fact, suggests strong destabilisation when $E_{22} \rightarrow 0^{-}$. In this case, even a "cylindrically" stable $2 / 1$ mode may be destabilised by mode coupling.

The effect of mode coupling was previously tested numerically by Fitzpatrick [21] with the T7 stability code. The T7 code, however, assumes very small inverse aspect ratio $(\varepsilon<0.15)$, elongation and triangularity and is, therefore, not directly applicable to the TCV experiments. Moreover, only model parabolic pressure and current profiles are employed to solve the MHD equations.

In this article, we test the effect of toroidal coupling by calculating the coefficients $E_{i j}$ using equilibria reconstructed from the experimental data of TCV ramp-up discharges at a standard aspect ratio. PEST-3 is, in principle, able to calculate the complete matrix $\mathbf{E}$ for an arbitrary number of rational surfaces and arbitrary axisymmetric toroidal geometry. Unfortunately, since in these plasmas $q_{a} \sim 3$, the $q=3$ surface is very close to the plasma edge and the finite element scheme in PEST-3 does not show the typical linear convergence, thus leading to unreliable results. We therefore use PEST-3 to calculate the first diagonal element, $E_{11}$, and compute $E_{22}$ in the cylindrical approximation with the code DELTAPCYL [26].

An example of the $\Delta^{\prime}$ calculation as a function of $q_{a}$ is shown in figure 12 for a quasicircular plasma. It can be seen that $\Delta_{m=2}^{\prime}$ is always negative, indicating an intrinsic stability of the $2 / 1$ tearing mode as previously discussed. The free boundary $3 / 1$ tearing mode evolves from stable (negative $\Delta^{\prime}$ ) to unstable (positive $\Delta^{\prime}$ ) during the ramp-up. According to equation 4, when the $m=3$ mode crosses the marginal stability during the current rise, a strong destabilisation of the $2 / 1$ tearing occurs which may abruptly grow at $q_{a} \lesssim 3.6$, close to the experimental value $q_{a}=3.3$ at which the $2 / 1$ mode is observed to grow. We would like to stress here that the rather peculiar conditions required for this destabilising coupling to occur are well met in the experiment. This, together with calculations shown above, indicates that the toroidal mode coupling destabilises the 2/1 tearing mode during the plasma current rise in TCV.

We note that, strictly speaking, the equations 3 and 4 are valid only for circular 


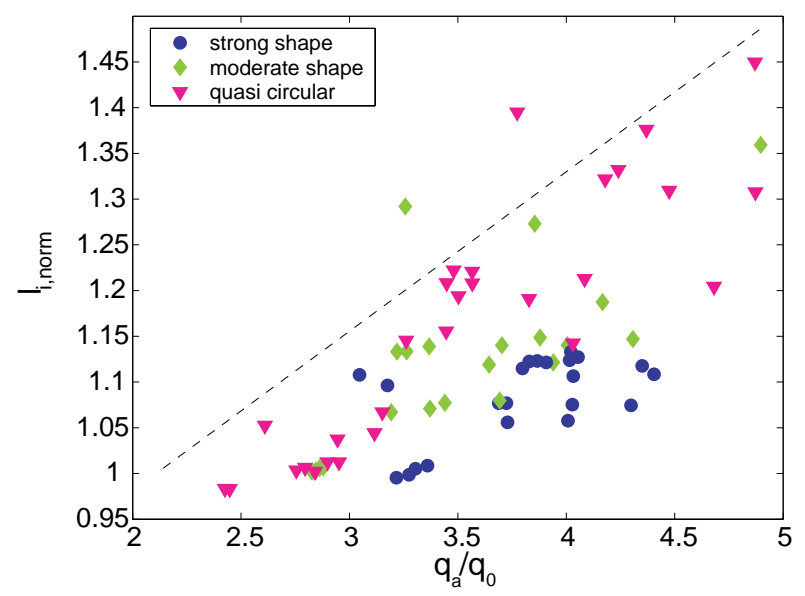

Figure 13. The normalised internal inductance is plotted against the $q$-profile peaking factor which shows, for a given value of $q_{a} / q_{0}$, the tendency toward a lower $l_{\mathrm{i} \text {,norm }}$ with increasing plasma shape for $q_{a} \sim 3$.

plasmas. The presence of a shaped cross-section introduces several, potentially important, effects on stability. Some of them are considered in the next section.

\subsection{Plasma shape stabilisation of the 2/1 tearing mode during current rise}

In order to understand the plasma shape stabilisation, we identify three potentially stabilising effects: 1) the change in the plasma free energy due to any modification of the equilibrium (particularly in the current profile), 2) the stabilising effect of the conducting walls, and 3) the coupling of the $q=2$ resonant surface to vacuum rational surfaces. The variation of the plasma $\beta$ with shape is relatively small in these experiments and while its effect is retained in the $\Delta^{\prime}$ calculation with PEST-3 it is otherwise neglected (i.e. GGJ term [27] or bootstrap current contribution).

1) We have analysed the experimentally reconstructed current profiles in terms of global profile peaking indicators, such as the $q$-profile peaking factor, $q_{a} / q_{0}$, and the normalised internal inductance, $l_{i, n o r m}$ [28]. The normalised internal inductance is a more reliable current peaking indicator than the standard internal inductance in experiments with variably shaped plasmas, since it is basically shape independent. We find that increasing the plasma shape, generally broadens the current profile, as it can be seen in figure 13. For an easier direct comparison, we select few discharges with the similar edge safety factor, plasma density and edge magnetic structure but different shape and profile peaking. From the calculations, to the broader current profile of shaped plasmas does not correspond an improved tearing stability. In contrast with the experimental observations, $r_{s} \Delta^{\prime}$ often increases with the shaping as showed in table 1. Similarly, the diagram in figure 10 predicts lower stability with the flattening of the current profile. In our case, however, the effect of the current profile broadening is mixed with the effect of the plasma shape. To further search for any intrinsic effect of the shaped cross-section on stability, we calculate the $\Delta^{\prime}$ for a set of equilibria with different values of $\kappa$ and $\delta$ 
Table 1. The $2 / 1$ tearing stability factor from PEST-3 code, $r_{s} \Delta^{\prime}$, for current rise discharges with $q_{a}=3.2$ and different plasma shaping. Note that the stability factor, calculated with the free boundary condition, has the lowest value for quasi-circular plasma and tends to increase with shaping, especially at negative $\delta$. The normalised internal inductance $l_{i, \text { norm }}$, the radial position of the $q=2$ surface, $\rho_{s}$, and $q_{0}$ are taken from LIUQE.

\begin{tabular}{|c|c|c|c|c|c|c|c|}
\hline shot\# & $r_{s} \Delta^{\prime}$ & $\kappa$ & $\delta$ & $l_{i, \text { norm }}$ & $\rho_{s}$ & $q_{0}$ & disruption \\
\hline 21400 & -7.2 & 1.3 & 0.2 & 1.19 & 0.90 & 0.76 & Yes \\
21395 & -3.1 & 1.4 & 0.2 & 1.15 & 0.91 & 0.80 & No \\
21399 & -0.2 & 1.7 & 0.2 & 1.06 & 0.92 & 0.88 & No \\
19405 & 3.5 & 2.5 & 3.5 & 0.91 & 0.90 & 0.74 & No \\
21402 & 11.1 & 1.3 & -0.4 & 1.11 & 0.85 & 1.10 & No \\
\hline
\end{tabular}

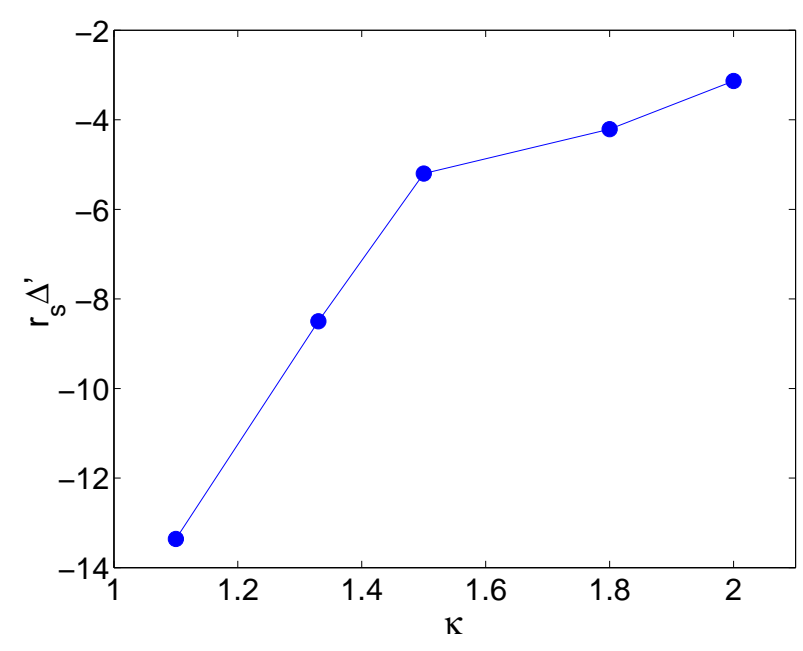

Figure 14. Tearing stability parameter $r_{s} \Delta^{\prime}$ as function of plasma elongation with fixed current profile shape (see text). The equilibria are re-scaled by fixing $q_{0}=0.75$.

keeping the same normalised current profile $j(\rho) / j_{\text {Max }}$ and pressure profile $p(\rho)$ as for discharge \#21400 (see table 1). Again, we find a negative trend in the stability given by the $\Delta^{\prime}$ with the plasma shaping, as shown for a $\kappa$-scan in figure 14. It appears that $\Delta^{\prime}$ calculations for single mode can hardly provides any guidance for the understanding of the plasma shape stabilisation observed in TCV.

2) The presence of the metallic wall may significantly change the tearing stability and, in particular, the effect of toroidal coupling (section 3.1). Although a perfect conductor at the plasma boundary does not dramatically improve the stability of an isolated internal mode (i.e. the $\Delta^{\prime}$ of the $m=2$ mode decreases by $20-50 \%$ ) it, however, easily stabilises surface modes close to the plasma edge (figure 15). This is true for any MHD mode resonant on a surface sufficiently close to perfectly conducting walls (see for instance figure 34 of ref. [18]). As a consequence, according to equation (4), no destabilising toroidal coupling occurs with a perfect conductor at the plasma boundary. 
In TCV, there is a minimum gap of few centimetres between the last closed flux surface and the metallic wall. The average gap distance changes strongly with the plasma shape. The boundary of a quasi-circular TCV plasma is on average relatively far from the vacuum vessel and therefore, a value of the stability index close to the free b.c. value is to be expected (i.e. the arbitrary full line in fig. 15). At large values of elongation and triangularity, however, the average distance between the plasma boundary and the wall decreases (see figure1), thus the external mode may be stabilised by the wall (dashed line in fig. 15) and the toroidal coupling inhibited. We suggest that this mechanism may be important in TCV and it could explain the plasma shape stabilisation. To demonstrate this, however, more theoretical and experimental work is needed. In particular, one would have to include in the calculations the exact geometry of the vacuum vessel, the plasma shape and the finite conductivity of the metallic walls.

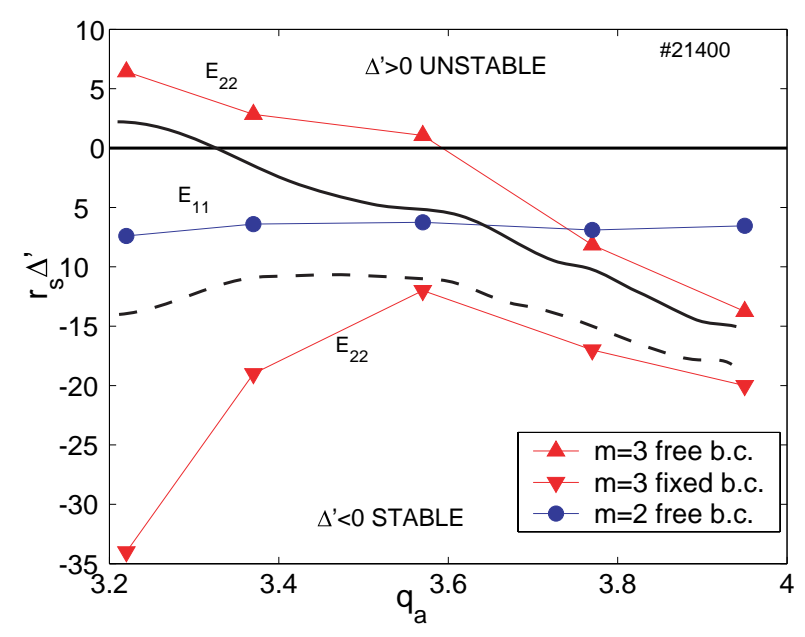

Figure 15. Tearing stability diagram for $m=2$ mode (circles) and $m=3$ modes with free (upward triangles) and fixed (downward triangles) boundary conditions for a "low shaping" case $(\kappa \simeq 1.3$ and $\delta \simeq 0.2$ ) from figure 12 , The solid and dashed black lines represent plausible but completely arbitrary paths in the tearing stability diagram when the real TCV-wall geometry is taken into account for a quasi-circular plasma and for a strongly shaped plasma respectively (see text).

3) Finally, mode coupling due to the plasma cross-section shaping is expected to influence the $2 / 1$ tearing mode stability. Pure toroidal geometry couples, in the lowest order approximation, to the $m \pm 1$ sideband of the principal $m$ poloidal component, resulting in the interaction of the $q=1,2$ and 3 rational surfaces, whereas elongation and triangularity couple the $m \pm 2$ and $m \pm 3$ harmonics respectively [21]. The $q=2$ tearing mode is thus influenced by the $q=4$ and $q=5$ resonance surfaces. In limited discharges, the $q=4$ and $q=5$ flux surfaces are located in the $2.5 \mathrm{~cm}$ of vacuum (minimum gap in the equatorial plane) that separates the plasma boundary from the conducting wall, and may therefore contribute to the global plasma stability through mode coupling. It has been shown numerically by Fitzpatrick [21] that both elongation and triangularity can increase the stability of the $2 / 1$ tearing mode for plasmas with 
$q_{a}<4$. However, further theoretical and numerical work is needed to extend these mode coupling results to plasmas with larger values of inverse aspect ratio, elongation and triangularity relevant to the TCV case.

\section{Summary and conclusions}

In current rise experiments, MHD instabilities appear for edge safety factors approaching low order rational numbers, like 5, 4, 3 and 2.5. We have analysed these instabilities in TCV. We find that the initial instability has dominant mode numbers $m / n=q_{a}$ and is thus localised at the plasma boundary, as commonly observed in many tokamaks, suggesting a link with the external kink mode.

In a quasi-circular plasma, this MHD activity often leads to plasma disruption when $q_{a} \sim 3$. The disruption is preceded by the fast growth of coupled $2 / 1$ and $3 / 2$ tearing modes accompanied by a decrease of the modes frequency due to the interaction with the conducting wall. Experiments with slow current rise rates in presence of sawtooth activity and peaked current profile are also disruptive in case of quasi-circular cross section. This excludes the role of transient effects, such as the destabilising skin-current effect.

We have also reported experimental evidences of destabilising mode coupling between the $3 / 1$ kink mode and the $2 / 1$ tearing mode in the initial phase of the MHD activity:

(i) the fast growth of the $2 / 1$ mode occurs at $q_{a} \lesssim 3$ (figure 4);

(ii) disruptions always occur when the $2 / 1$ tearing mode coexists with a $3 / 1$ mode rotating at the same frequency (figure 5);

(iii) disruptions do not usually occur if the $3 / 1$ and $2 / 1$ modes do not coexist or coexist but rotate with different frequency (figure 9).

The locking of the $2 / 1$ mode to the $3 / 1$ correlates with whether or not the $2 / 1$ becomes disruptive.

In TCV, moderate and strong plasma shaping stabilises the disruptive mode. Both plasma elongation $(\kappa>1.35)$ and positive $(\delta \gtrsim 0.2)$ or negative $(\delta \lesssim-0.3)$ triangularity prevent the growth of large $2 / 1$ magnetic islands. For strongly shaped plasmas, even the $3 / 1$ surface mode is absent. This positive effect of the plasma shaping is regularly used in TCV to achieve plasmas with $q_{a}<3$.

Calculations of the tearing stability index, $\Delta^{\prime}$, using the full geometry code PEST-3 and the TCV experimental profiles confirm the importance of mode coupling. In the current ramp scenario, the stability equations of coupled modes (equations (3) and (44)) successfully predict the destabilisation of an intrinsically stable $2 / 1$ tearing mode by the toroidal coupling effect, as suggested by Fitzpatrick [19].

Analysis of reconstructed and modelled TCV equilibria of shaped plasma have shown no correlation between the positive effect of plasma shaping in the experiment and the value of the tearing stability index. On the contrary, a destabilising trend with 
plasma shape is often observed in the $\Delta^{\prime}$ values. This seems to lead to the conclusion that single-mode $\Delta^{\prime}$ theory does not provide a good description of the effect of plasma shaping on tearing stability. However, some basic physics is missing in the present analysis. For instance:

(i) the effect of the conducting wall that strongly depends on the plasma shape and may be able to inhibit the destabilising toroidal coupling (figure 15). Unfortunately, a quantitative realistic modelling of the wall effect requires the implementation of the exact geometry of the TCV vacuum vessel into a numerical outer layer code such as PEST-3, and is beyond the scope of this work. Our results, however, suggest that the wall stabilising mechanism could be experimentally tested using the flexible shape and position control system of the TCV tokamak by varying the vertical position and/or the minor radius of the plasma discharge.

(ii) the effect of mode coupling with vacuum flux surfaces, such as $q=4$ and $q=5$. A theory which includes this coupling for plasma parameters relevant to the TCV case is still missing.

Since these two stabilising mechanisms act simultaneously and independently, more experimental and theoretical effort is needed to determine their respective role. It is important, however, to determine which mechanism is dominant: the effect of the conducting wall, perhaps more peculiar to the TCV geometry, or the mode coupling due to plasma shape, which is a more universal property of the MHD stability and may be used in other tokamaks to mitigate or suppress plasma disruptions.

\section{References}

[1] J. A. Wesson et al., Nuclear Fusion 29 (4) (1989) 641.

[2] Z.A. Pietrzyk et al., Nuclear Fusion 32 (10) (1992) 5.

[3] A. D. Cheetham et al., Nuclear Fusion 27 (5) (1987) 693.

[4] F. Alladio et al., Nuclear Fusion 26 (1986) 11-22.

[5] S. Turlur, Ph.D. thesis, EUR-CEA-FC-1559, CEN Cadarache, France (1997).

[6] H. P. Furth et al., Phys. of Fluids 6 (1963) 459-484.

[7] F. Hofmann et al., Plasma Physics and Controlled Fusion 36 (12B) (1994) B277-B287.

[8] F. Hofmann, G. Tonetti, Nuclear Fusion 28 (10) (1988) 1871.

[9] C. Angioni, Private communication (2002).

[10] M. Schittenhelm, H. Zohm, Nuclear Fusion 37 (9) (1997) 1255-1270.

[11] H. Reimerdes, Ph.D. thesis, Ecole Polytechnique Fédérale de Lausanne, thèse n.2399 (2001).

[12] G. Bateman, MIT Press, Cambridge, Massachusetts, USA, 1978.

[13] M. Nave, J. Wesson, Nuclear Fusion 30 (12) (1990) 2575-2583.

[14] W. A. Waddell et al., Phys. Rev. Lett. 41 (20) (1978) 1386-1389.

[15] R.M. Coelho et al., Physics of Plasmas 6 (4) (1999) 1194-1202.

[16] H. Reimerdes, A. Pochelon, O. Sauter et al., Plasma Phys. Control. Fusion 42 (2000) 629-639.

[17] A. Martynov, J. P. Graves and O. Sauter, Plasma Physics and Controlled Fusion 47 (10) (2005) 1743-1762.

[18] J. A. Wesson, Nuclear Fusion 18 (1978) 87.

[19] R. Fitzpatrick, R. Hastie, T. Martin, C. Roach, Nuclear Fusion 33 (10) (1993) 1533.

[20] M.F.F. Nave et al., Eur. Phys. J.D 8 (2000) 287. 
[21] R. Fitzpatrick et al., Nuclear Fusion 33 (10) (1993) 1533-76.

[22] S.C. Cowley and R.J. Hastie, Phys. Fluids 31 (3) (1988) 426.

[23] A. Pletzer, A. Bondeson, R. L. Dewar, Journal of Computational Physics 115 (3) (1994) 530.

[24] D. P. Brennan, E. J. Strait, A. D. Turnbull, M. S. Chu, R. J. L. Haye, T. C. Luce, T. S. Taylor, S. Kruger, A. Pletzer, Physics of Plasmas 9 (7) (2002) 2998-3006.

[25] M. S. Chu, R. J. L. Haye, M. E. Austin, L. L. Lao, E. A. Lazarus, A. Pletzer, C. Ren, E. J. Strait, T. S. Taylor, F. L. Waelbroeck, Physics of Plasmas 9 (11) (2002) 4584-4590.

[26] M. S. Chu, Private communication (1994).

[27] A. H. Glasser, J. M. Greene, and J. L. Johnson, Phys. Fluid 18 (1975) 875.

[28] L. Lao and et al., Nuclear Fusion 25 (1985) 1321.

\section{Acknowledgments}

The authors would like to acknowledge fruitful discussions with O. Sauter and E. Lazzaro. This work was partly supported by the Swiss National Science Foundation. 